\title{
The Design of Network-based Virtual Laboratory
}

\author{
Li Yong ${ }^{1, ~ a ~, ~ L i ~ G u a n g m i n g ~}{ }^{2, b}$ \\ ${ }^{1}$ Electrical \& Information Engineering College Shaanxi University of Science and Technology,Xi'an, \\ Shaanxi, China \\ ${ }^{2}$ Electrical \& Information Engineering College Shaanxi University of Science and Technology,Xi'an, \\ Shaanxi, China
}

aliyong@sust.edu.cn, 'liguangming@sust.edu.cn

Keywords: Virtual laboratory, virtual instrument, construction, structure design

\begin{abstract}
Virtual laboratory is a practical teaching assisted instruction with high efficiency. This paper analyzes the characteristics, classification of virtual laboratory and the design of virtual instrument. It discusses the construction and system structure of network-based virtual laboratory. This paper also proposes design method of hardware and software, and provides a reference for the construction of virtual laboratory.
\end{abstract}

\section{Preface}

The famous American national instrument company (NI), first proposed the concept of software as an instrument of virtual instrument in the mid - 1980s[1]. Virtual instrument is a kind of equipment which combines the hardware resources and the actual equipment together into a whole through the application of software, and completes the data display, data analysis processing and other functions through the software. Virtual laboratory is based on the technical support of computer hardware and software to realize the virtual environment and the virtual experimental methods[2]. The real experimental environment and equipment and the experimental process that the virtual experiment reproduced ,provided the experimenter very real feeling, and it is easy to use, it has high efficiency, and not be limited by time and place, etc.The application of virtual laboratory allows students the freedom to experiment and realize data sharing.

For the courses in the Science and Engineering Colleges or Universities, the experimental teaching is very important. The existing experiment teaching content and method have great limitations[3]. There are some problems, such as time-consuming in management, decentralization of funds, backward equipment, low utilization of the laboratory, restriction of students' practice, etc. Virtual laboratory[1] can solve the problem effectively. In recent years, virtual experiment has become a product of information technology and computer network technology, it is the important means to improve science experiment mode and method[4], and it will be very helpful to improve the quality of experiment teaching. some universities and research institutions have established a certain scale specialized virtual laboratory, and received good effect. The construction and popularization of university virtual laboratory has become an inexorable trend of the future development.

\section{The classification and characteristics of virtual laboratory}

Comparing to the theoretical teaching, experimental teaching is more intuitive and practice. It plays an important role in enhancing the quality of education and develop innovative ability of the students. Virtual laboratory is a new science research method based on computer virtual system[5], It put the computer technology and traditional instruments technology together[6], It is based on computer network, and expand the function of traditional experiment instrument. It realizes data collection, analysis and operation through the network's connection with virtual instrument equipment.

There are three common ways of virtual experiment in virtual laboratory. 
a) Remote control experiment: The real experimental equipment and control systems are connected to the terminal by using the computer technology and communication technology. The experimenter can operate his own computer through the computer network. They can do the experiment with remote control real equipment, as well as the real-time observation[7].

b) Simulation experiment: it is displayed as text, graphics, or animation by using computer software simulates experiment conditions, experimental equipment, instruments and components, etc. During this process, the experimenter does the experiment by interacting with the experimental software.

c) Virtual experiment: it builds a virtual environment, virtual instruments and virtual device using computer technology and virtual technology and it can provide a realistic simulation environment for the experimenter. The experimenter has the feeling of doing the experiment in the real laboratory.

The characteristics of virtual laboratory:

a) Very good degree of simulation. The function and operation of the virtual instrument and real instrument are the same in virtual laboratory system[8]. It is as convenient as in real laboratory to do experiments in the virtual laboratory. The experimenter can obtain the good effect of learning.

b) Free time and space of experiment. Compared with the traditional experimental mode, there is no place and time limit for virtual experiment. The experimenter can do the experiment in any time and any place. The experimenter can establish his own way of using virtual instrument and process and preserve their own experimental data and material.

c) Reduce the cost effectively. Virtual laboratory can use repeatedly, and there is no experimental equipment consumption, wear, destroying problems. It can save maintenance cost and avoid repetition purchase of the instruments and the equipment,

d) Good scalability. According to the characteristics of virtual laboratory, virtual experiment can expand the scope and subjects. Virtual laboratory equipment and instruments[4] can complete varieties, and easy to upgrade and add new varieties. It can effectively solve the problem that can affect the experiment because of the incompleteness of experimental instruments.

e) Good collaboration. By using the network technology and facilities, and the collaborative virtual environment technology, the experimenter can do alliance cooperation and collaborative research with remote experimenter. It can provide distributed research environment for the researchers who are engaged in the same project research in different places.

Virtual laboratory effectively improves the practical teaching efficiency through the computer and network technology[9]. It develops the experimenter's ability in analysis and problem solving. It also improves the experimenter's practical skills, and makes high-tech in the education field fully play the advantages[10].

\section{The structure and design of virtual laboratory based on the WEB}

Virtual laboratory construction is to establish a special website that can control the virtual instrument and operation of virtual experiment or remote control experiment instrument based on the web[11]. It includes computer software technology, the virtual instrument technology, electronic instrument technology, network technology, database technology and communication technology, etc[12]. Common network-based virtual laboratory is composed by virtual experiment and software simulation equipment, and virtual experiments can be divided into simulation experiment and remote control experiment. Simulation experiment is done by using software simulates the environment[13]. Remote control experiment is that experimenter control the real experiment equipment to do the experiment by the network. Virtual laboratory system make a virtual laboratory have a broader and more adaptable applications using a combination of both[14]. As shown in figure 1 


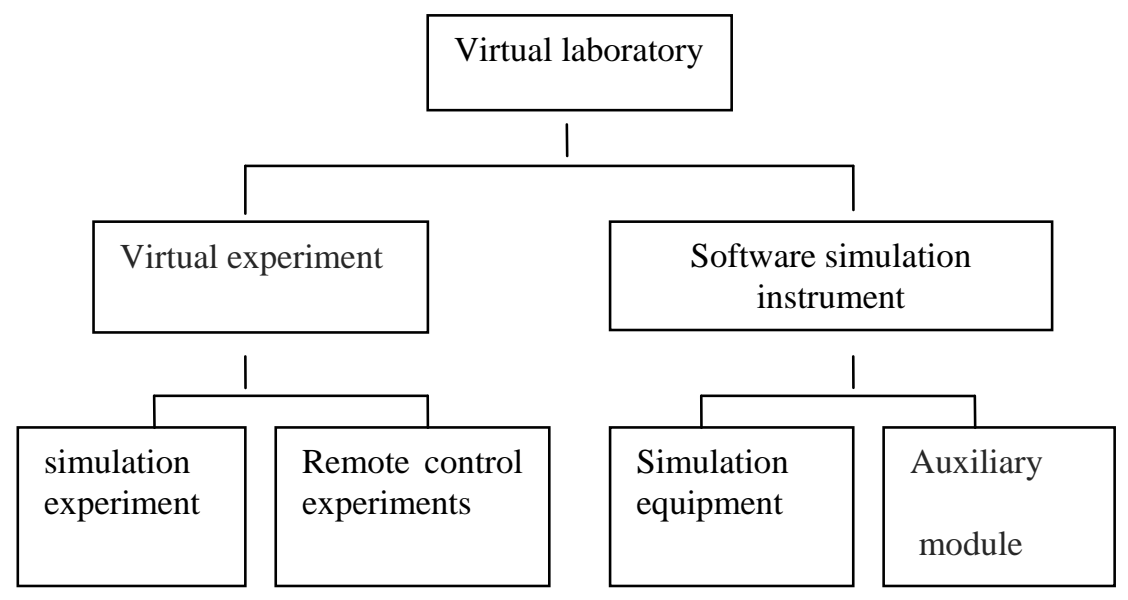

Fig.1 Structure of virtual laboratory

It often uses BSDA (Browser/Server/database\&application) structure to build remote control experiment. In such a structure, application server mainly controls experiment instrument, collecting and processing experimental data and send the experimental data to the web server[15]. Data transmission process, there is a corresponding protection and verification measures, to ensure the accuracy of data transmission.Web server to ensure the validity and the logged on user order,at the same time provides user authentication management and open interactive experimental environment. The database plays the role of managing user's information, producing a dynamic web[16], and storing and managing the laboratory resources. The client's main function is to realize the remote control of the virtual instrument for the user, and to provide convenient and reliable service for users. As shown in figure 2

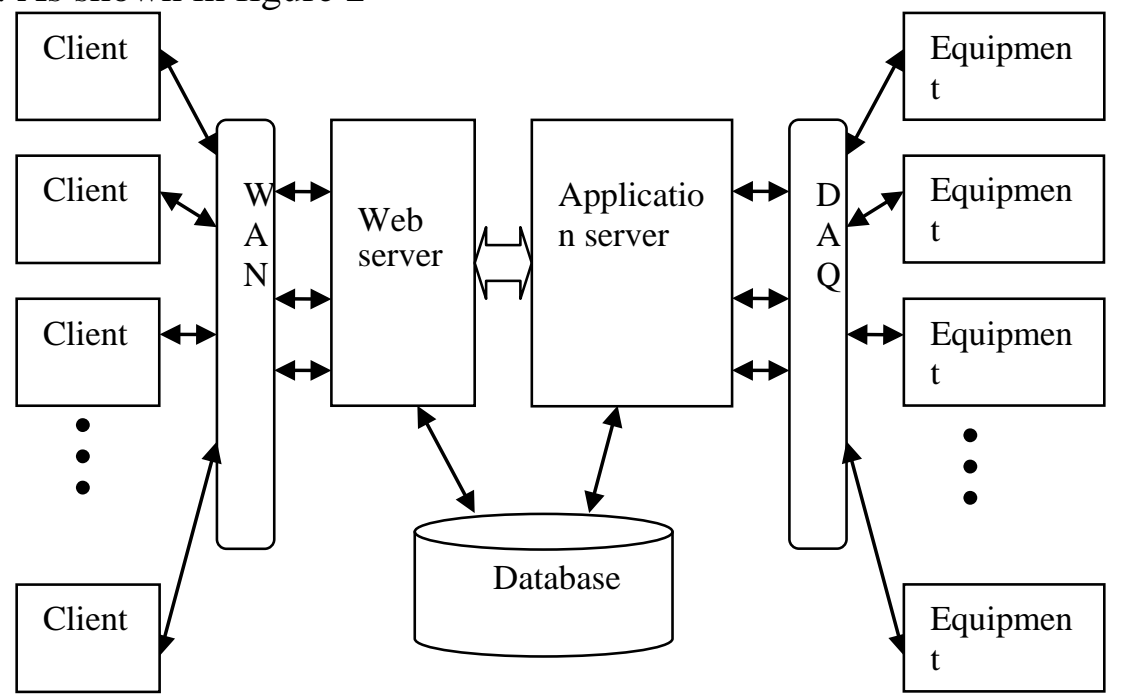

Fig.2 Structure of remote control experiment

Virtual instrument is an important part of virtual experiment. Virtual instrument includes hardware and software. The main function of the hardware is to obtain the true test signal in real experiment, and the function of software is to control the data collection[17], analysis and processing, and keep them integrate in the instrument control environment.

User can use the Browser to access the Server directly and easily. The update of experimental program and maintains be finished by the Server. If the Users can connect to the Internet, they can access the lab servers by the client's browser too. Different users can use the experiments at the same time[18]. Test server provides access to the client browser service, it's providing services such as open interaction environment, etc. Instrument control server is responsible for data collection, statistics and management. The database of server can share the experimental data and the experimental methods and rules.Common virtual instrument structure as shown in figure 3 


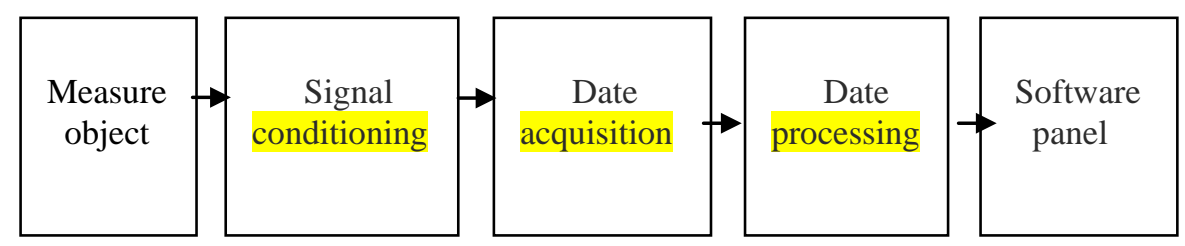

Fig.3 Formation of virtual instrument

It often uses the software LabVIEW of National Instruments(NI) to develop the virtual instrument. LabVIEW is a graphical programming language based on the test system software development platform. Its network communication function is very good. It can use Remote Panels technology to realize the remote data acquisition control. The ActiveX function of LabVIEW can access to the database. All the LabVIEW application is virtual instrument[19].

Simulation experiment is mainly for the verification experiment. Virtual laboratory system can use Browser\&NIWeb Server structure, design for the Web and generate embedded experimental platform Web pages in the Web server through the LabVIEW built-in web publishing function.

Users can access the virtual laboratory in the far terminal through the Web browser.And to do the virtual experiment that they need. The working principle of the user access to the virtual experiment as shown in figure 4

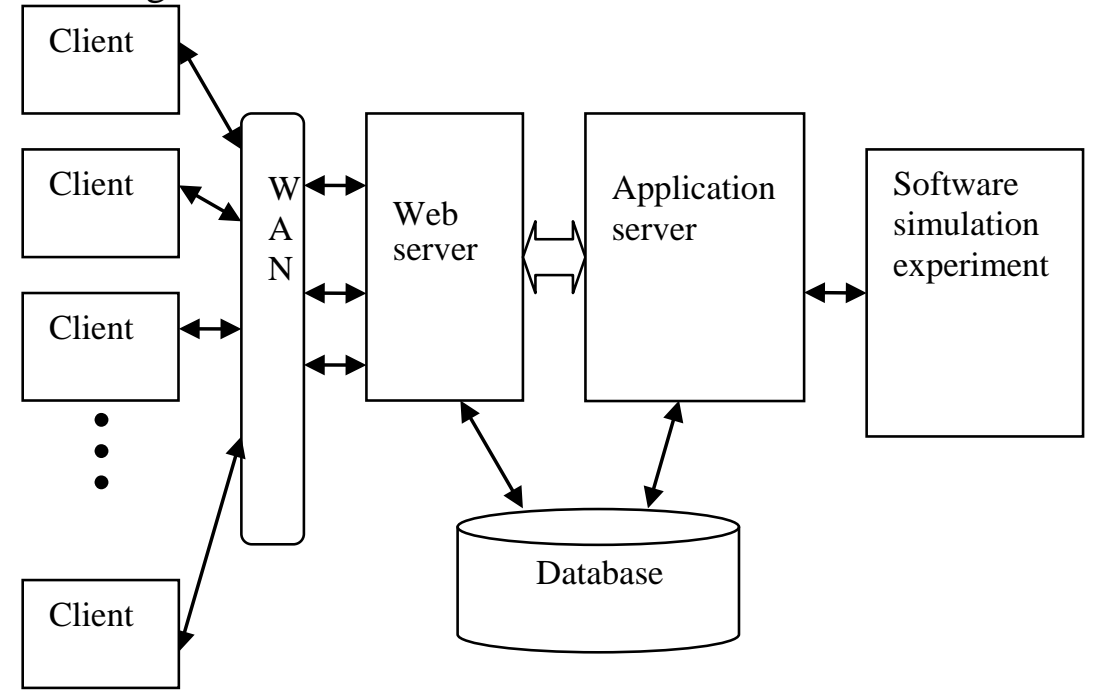

Fig.4 Structure of simulation experiments

The realization of the virtual laboratory, in addition to the above hardware, server and transmission lines, but also the need for a corresponding configuration, for each client and server these configurations, mainly refers to the function of software, only the configuration of software, can make the hardware really play its function, virtual experiment system can really be normal operation, and to achieve its intended function[20].

Its software structure as shown in figure 5 


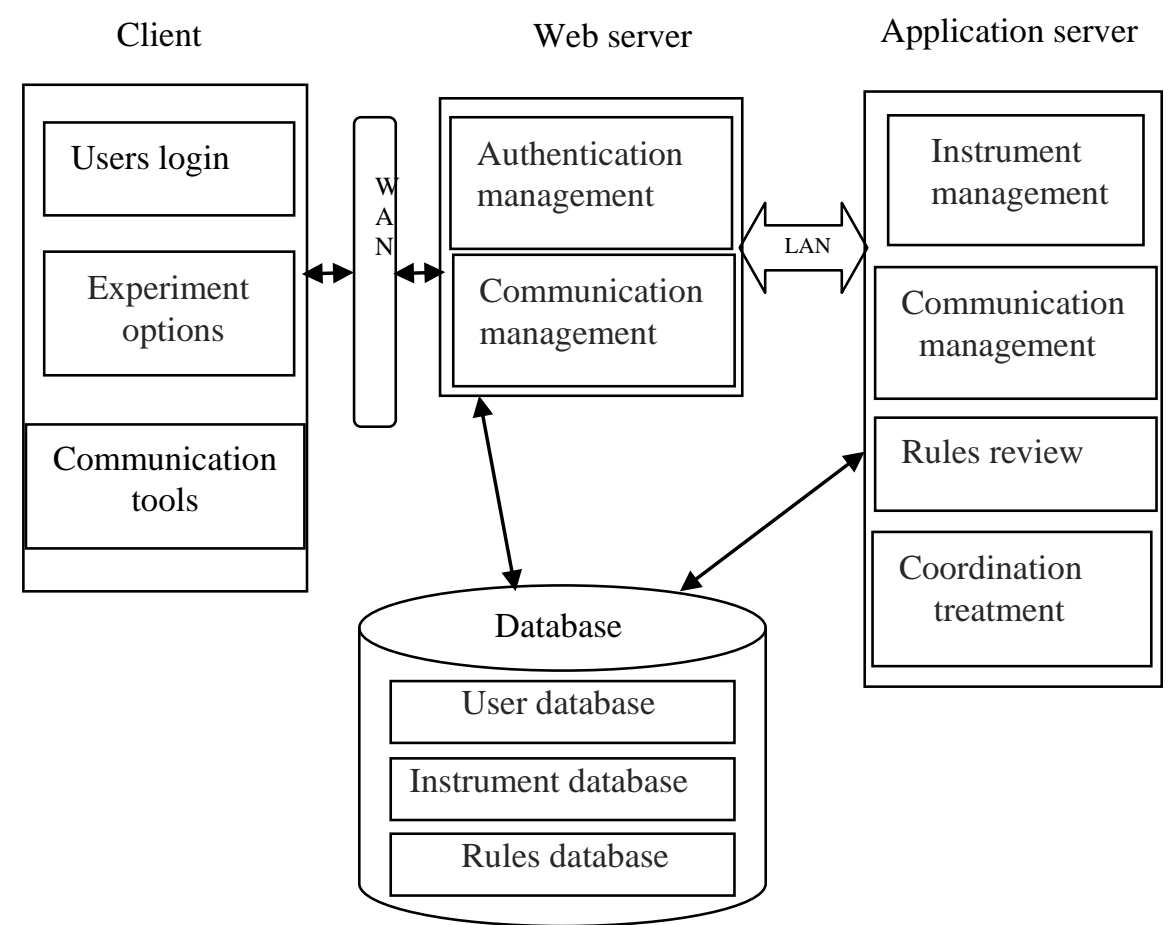

Fig.5 Structure of software

\section{Summary}

Virtual laboratory is a good assistance for experiment teaching. And it will become the development direction of the experiment teaching in the future.It has good practicability and great development potential.

This paper discusses the virtual laboratory types and application of occasions, and gives several methods to construct the virtual laboratory.And discusses the construction of the mode in detail, this paper can provide effective help and reference for the construction of Virtual Laboratory.

Virtual technology has entered a relatively mature stage of development.And applications in various fields has made the important position. In the application of virtual lab, it also plays an important role, the virtual experimental advantages will enable the rapid development of application of virtual lab.It will play a considerable role in the modern practice of education. As a new means of experiment, the virtual laboratory experiments will provide convenience service for more users, open up a new road for the experimental subject.

\section{References}

[1] Margery Weinstein. A Better Blend[J]. Training,2010, (9): 32-34.

[2]Ji-Ping Zhang. Hybrid Learning and Ubiquitous Learning[A]. ICHL'08: Proceedings of the 1st international conference on Hybrid Learning and Education[C] . Hong Kong , China,2008.

[3] Steyskal H, and Schindler J K. Separable space-time pattern synthesis for the techsat21 space based radar system [J].IEEE Proceedings Aerospace conference.2006(2):955-960.

[4]Texas Instruments. SimpliciTL low-power RF protocol [M].Dallas:Texas Instruments, 2010.

[5] National Instrument, Educational Laboratory Virtual Instrumentation Suite, Austin:NI, 2011.

[6]Davis M E. Space Based Radar Moving Target Detection Challenges [J].IEE Conference Publication,n490, 2002,2921-2924.

[7]Depren,O, An intelligent intrusion detection system for anomaly and misuse detection in computer networks, Expert System with Applications, vol 29,pp. 713-722, 2005.

[8]Texas Instruments, SimpliciTL low-power RF protocol, Dallas:Texas Instruments, 2009. 
[9]Nitin Swamy, Internet-based educational control system lab using net meeting, Transaction on Education, vol.45, pp. 145-150, 2011.

[10] National instruments. The Measurement and Automation Catalog 2009, 49-137

[11] Zhou H,Wang L.2010. Virtual instrument system software architecture description language.Jourmal of Chinese Zhejiang University(SCIENCE), 2(4):411-415

[12] MJ. Callaghan, J. Harkin, C. Peters, et al. A Collaborative Environment for Remote Experimentation. IEEE International Conference on Microelectronic Systems Education, 2011, $62 \sim 63$

[13] John L.Watson, George Bibel, Kenneth Ebeling, et al. On-line laboratories for undergraduate distance engineering students.34th Annual Frontiers in Education Conference, 2009, T3C/1 T3C/6

[14] Heinz-Dietrich Wuttke, Karsten Henke, Nadine Ludwig. Remote Labs versus Virtual Labs for Teaching Digital System Design. International Conference on Computer Systems and Technologies, 2012, IV.2-1 IV2-6

[15] Ulrich Heinkel, Martin Padeffke, Oliver Kraus, et al. Virtual-Classroom: An Interactive Teaching System for the Design of Integrated Circuits. Global Journal of Engineering Education, 2008, 1(2):17

[16] J. A. del Alamo, V Chang, L. Brooks, et al. The MIT Microelectronics WebLab: a Web-Enabled Remote Laboratory for Microelectronic Device Characterization.World Congress on Networked Learning in a Global Environment, 2012, http://weblab.mit.edu

[17] Luis Anido, Martin Llamas, Manuel J. Fernandez. Internet-based Learning by Doing. IEEE Translation on Education, 2011, 44(2):18

[18] Marco Casini, Domenico Prattichizzo, Antonio Vicino. The Automatic Control Telelab: a Remote Control Engineering Laboratory. 80th IEEE Conference on Decision and Control, 2011, 3242 3247

[19] Chi Chung Ko, Ben M. Chen, Shaoyan Hu. A Web-Based Virtual Laboratory on a Frequency Modulation Experiment. IEEE Translation on Systems, Man, and Cybernetics, 2011, 31(3):297 303

[20] Nihnja K. Swain, James A. Anderson, Ajit Singh, Mrutyunjaya Swain, Marvin Fullon, Joseph Garrett, Omaria Tucker. Remote Data Acquisition, Control and Analysis using LabVIEW Front Panel and Real Time Engine. Proceedings IEEE SoutheastCon 2012. 